\title{
Profiling of Histidine Phosphorylation in C2C12 Mouse Myoblast Cell Line
}

\author{
YoSHIKI MIURA*, SAIKO KAZUNO*, HIKARI TAKA*, TAKASHI UENO* \\ * Laboratory of Proteomics and Biomolecular Science, Research Support Center, Juntendo University Graduate School of \\ Medicine, Tokyo, Japan
}

Protein phosphorylation is one of the most common and well-studied post translational modification in eukaryotic and prokaryotic cells. Among phosphoamino acid, phosphohistidine (pHis) is mainly investigated in microorganisms and plants. However pHis containing proteins existed in vertebrates (especially in mammals), such as histone $\mathrm{H} 4$, trimeric G protein $\beta_{1}$ chain, potassium-calcium channel KCa3.1, studies on invertebrates were limited. In contrast to acid-stable $O$-phosphorylation of Ser, Thr and Tyr residues, His residue forms heat and acid-liable phosphoramide bond, which is difficult to identify using conventional low $\mathrm{pH}$ reverse phased LC-MS/MS. In addition to its unstable nature, histidine residue has two phosphorylation sites on its imidazole nitrogen atoms (N1, N3), which complicates its analysis.

Recently T. Hunter and his colleagues developed antibodies against each N1-, N3-, specific pHis using stable each pHis analogues (Fuhs et al, 2015). To profile phosphorylation of histidine residue, we concentrated phosphohistidine containing peptides from tryptic digest of $\mathrm{C} 2 \mathrm{C} 12$ mouse myoblast cell lysate with anti-pHis antibodies and peptide sequences were determined with Triple TOF 5600 (SCIEX). Candidate of histidine phosphorylated proteins were mainly categorized into actin cytoskeleton signaling, glycolysis and DNA binding protein. To elucidate the roles of histidine phosphorylation, cDNA encoding pHis phosphatase-1 (PHPT-1) which specifically dephosphorylates pHis was transfected into $\mathrm{C} 2 \mathrm{C} 12$ cell. Quantitative analysis of immuno-purified peptides were performed with SWATH-MS, a data independent acquisition method, revealed peptides derived from glycolytic enzymes were markedly decreased in PHPT-1 transfected cell. These results raise the possibility that glycolysis would be regulated through histidine phosphorylation.

Key words: phosphohistidine, proteomics 